Ann. Sci. For., 1986, 43 (3), 365-382

\title{
Optimisation du sciage du pin maritime
}

\author{
A. BOUZINEKIS, P. MARTIN \\ C.R.A.N., U.A. $n^{\prime \prime} 821$ du C.N.R.S., E.S.S.T.I.B. \\ Université de Nancy 1, B.P. 239, 54506 Vandauvre-lès-Nancy Cedex
}

\section{Résumé}

Cette étude a été entreprise dans le cadre d'un contrat de recherche D.G.R.S.T. filière bois coordonné par le C.E.M.A.G.R.E.F., en vue de la sélection de jeuncs plants de pins maritimes (Pinus pinaster) des Landes.

Des simulations de débit ont été développées afin d'aider au choix entre les critères opposés de verticalité et de vigueur.

Dans lc mode d'exploitation actuel, les grumes sont débitées en billons de $2 \mathrm{~m}$ et de 2,6 $\mathrm{m}$ assimilés àt un tronc de cône.

Dans l'optique d'utilisation en charpente ou en ossature de maison, la simulation a porté sur l'obtention de produits de $6 \mathrm{~m}, 3 \mathrm{~m}, 2,6 \mathrm{~m}$ ou $2,4 \mathrm{~m}$. Dans ce cas, l'hypothèse morphologique précédente n'est plus valable et la forme des arbres a été modélisée par deux paraboles. Les programmes d'optimisation de débit ont permis de montrer que le rendement volumique est meilleur lorsque l'on tronçonne le premier billon à $3 \mathrm{~m}$, et qu'une corrélation entre le rendement matière et l'écart à la verticalité n'a pu être obtenue, mais d'autres corrélations ont été mise en évidence.

Mots clés : Sciage, optimisation du sciage, Pinus pinaster, grames courbes, bois de compression.

\section{Présentation de l'étude}

La nécessité de disposer d'éléments quantitatifs pour une aide à la sélection des individus et une meilleurs adaptation aux marchés futurs est la base d'un contrat de recherche financé par la D.G.R.S.T. sur le défaut de rectitude basale du tronc de pin maritime de provenance landaise (Pinus pinaster) et coordonné par le C.E.M.A.G.R.E.F. (Division Graines et Plants Forestiers, Domaine des Barres, 45290 Nogent-sur-Vernisson).

Dans ce cadre, nous avons développé une simulation du sciage des grumes de pin maritime afin d'étudier l'influence du défaut de verticalité sur le rendement volumique et financier après avoir modélisé la forme des arbres. 
Dans un premier temps, le C.P.F.A. (1) et le C.E.M.A.G.R.E.F. ont choisi un échantillonnage de 150 arbres dans un peuplement. Ces arbres ont été répartis en 6 classes suivant leur écart à la verticale, défaut mesuré à $1,50 \mathrm{~m}$ du sol du côté concave de l'arbre.

Chaque arbre de l'échantillon après avoir été numéroté, a été mesuré à trois hauteurs $(0 \mathrm{~m}, 1,50 \mathrm{~m}$ et $2,66 \mathrm{~m})$ pour obtenir leur défaut de courbure (côté convexe) par rapport à la verticale du niveau $5,32 \mathrm{~m}$. Ces arbres devaient manifester une seule courbure et être considérés comme étant constitués de deux billons : le premier (la "patte ") d'une longueur de $2,05 \mathrm{~m}$, destiné à être scié et le deuxième d'une longueur, soit de $2,05 \mathrm{~m}$ destiné à être scié, soit de $2,66 \mathrm{~m}$ destiné à être déroulé.

De chacune des classes ( 25 arbres), on tire au hasard 8 arbres, qui constituent le sous-échantillon, usinés sous la surveillance du C.T.B.A. (2) dans la Scierie DuDEZERT (3). Ainsi, pour chacun des billons du sous-échantillon, on était en mesure de connaître sa longueur ainsi que deux diamètres perpendiculaires et le périmètre sous écorce pour ses deux extrémités.

De plus, trois rondelles sont tirées des arbres du sous-échantillon après abattage et tronçonnage : la première au niveau 0 et les deux autres à l'extrémité de chaque billon. Sur chacune de ces rondelles ont été mesurées par le Laboratoire des Recherches sur les Produits Forestiers (4) la surface et la position du bois de compression, l'excentricité de la moelle, la position relative de la moelle par rapport à 8 points choisis sur le périmètre ainsi que l'épaisseur de l'écorce en ces points [7] (*).

A partir de ces éléments, nous avions à réaliser des logiciels de simulation de débit des grumes qui répondent aux objectifs suivants :

$1^{\circ}$ Déterminer le débit théorique optimal correspondant aux modes de sciage utilisés généralement en scierie ;

$2^{\circ}$ Trouver l'influence des particularités du pin maritime (courbure, bois de compression) sur le rendement volumique et financier.

Afin de simuler la découpe pour la charpente et les éléments de maisons à ossature en bois, ce dernier point implique une extrapolation des dimensions de grumes à une longueur de $6 \mathrm{~m}$ et une simulation du tronçonnage à des niveaux $2,40 \mathrm{~m}, 2,60 \mathrm{~m}$ et $3 \mathrm{~m}$ au lieu de $2,05 \mathrm{~m}$ actuellement.

Après avoir déterminé la forme réelle des arbres à partir des valeurs mesurées définie par deux fonctions paraboliques, un programme d'optimisation de celles-ci a été développé. Le logiciel détermine la section des produits principaux que l'on peut placer dans différentes partitions de la grume et les dimensions des sous-produits tirés des dosses.

Des programmes d'optimisation fondés sur une géométrie de la grume assimilée à un cylindre ou un tronc de cône ont été développés par différents laboratoires

(1) C.P.F.A., Centre de Productivité et d'Action Forestière d'Aquitaine, 17, rue Esprit-des-Lois, F 33080 Bordeaux Cedex.

(2) C.T.B.A., Centre Technique du Bois et de l'Ameublement, 10, avenue de Saint-Mandé, F 75012 Paris.

(3) Scieric Dudezert, Cabanac et Villagrains, F 33650 Labrède.

(4) Laboratoire de Recherches sur les Produits Forestiers, I.N.R.A.-C.R.F.-E.N.G.R.E.F., 14, rue Girardet, F 54042 Nancy Cedex.

(*) Les chiffres entre crochets carrés renvoient aux références bibliographiques. 
$[3,4,5,6,10,11]$; dans cette étude la forme particulière du pin maritime est prise en compte. L'introduction des caractéristiques technologiques avait été également envisagée mais les études effectuées par le Laboratoire des Recherches sur les Produits Forestiers n'ont pu déboucher sur une modélisation simple des zones de bois de compression. En effel la forme de ce bois de compression est trop irrégulière et sa répartition dans les différents niveaux de la tige est beaucoup moins stable qu'on pourrait l'imaginer d'après nos connaissances actuelles [7].

Les programmes ont été écrits en BASIC et sont exploitables sur micro-ordinateur (SIL'3 de Leanord, SIRIUS, ...), matériel qui peut être facilement acquis par une scierie.

\section{Madélisation des arbres}

Pour garder l'homogénéité de la représentation et compte tenu des données à notre disposition, nous avons adopté une forme d'équation du $2^{\mathrm{e}}$ degré [1].

Pour chaque arbre du sous-échantillon, on dispose des coordonnées de quatre points (fig. 1) de la partie convexe et les dimensions de deux billons qui sont déjà tirés (longueur du billon, deux diamètres perpendiculaires à chaque extrémité).

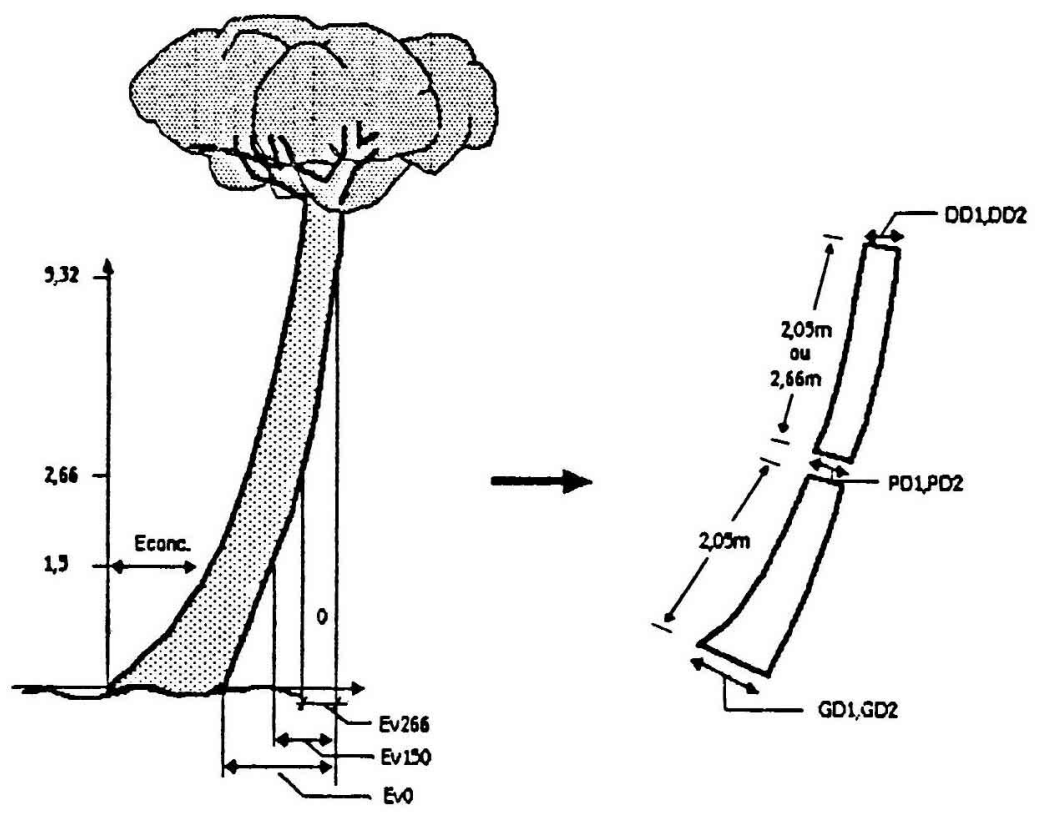

FIG. 1

Données sur les arbres du sous-échantillon.

Data of trees in the sub-sample. 
A partir de ces données, on détermine la courbe qui passe par ces quatre points : $F_{1}(x)=A_{1} x^{2}+B_{1} x+C_{1}$. Les arbres présentant une inflexion ne sont pas pris en compte car ils ne sont pas représentatifs (cadre du projet).

A l'aide des valeurs des longueurs des deux billons, on détermine les extrémités de ceux-ci sur le côté convexe (points $\mathrm{PB}(1), \mathrm{PC}(1)$ sur la figure 2). On mène les normales aux tangentes qui passent par ces trois points (extrémités des billons) et à l'aide de trois diamètres perpendiculaires, on trouve les coordonnées de trois points du milieu géométrique ( $\mathrm{PA}(2), \mathrm{PB}(2), \mathrm{PC}(2))$ et les trois points de la partie concave de l'arbre (PA(3), $\mathrm{PB}(3)$ et $\mathrm{PC}(3)$ sur la figure 2).

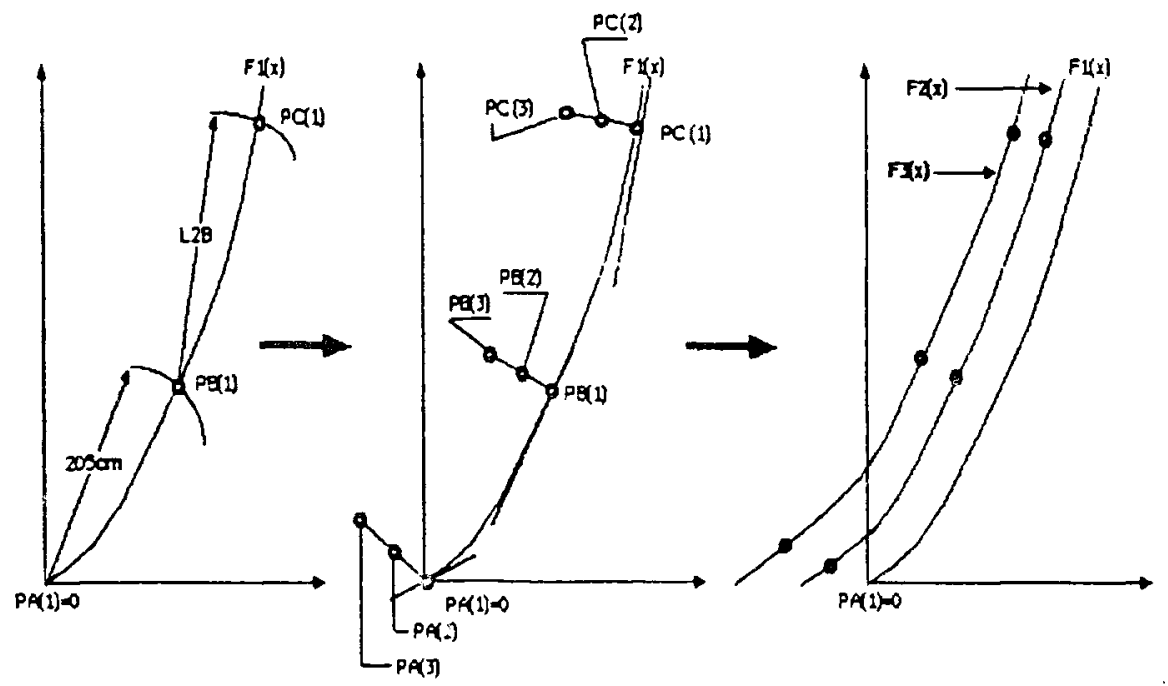

FIG. 2

Détermination des courbes $F 2(x), F 5(x)$.

Curves $F 2(x), F 3(x)$.

D'où les équations $F_{2.2}(x)=A_{2.2} x^{2}+B_{2.2} x+C_{2}$ de la ligne moyenne et $F_{3}(x)=A_{3} x^{2}+B_{3}+C_{3}$ de la partie concave. Les relations $F_{1}(x), F_{3}(x)$ et $F_{3}(x)$ fournissent une représentation de la grume, la section en tout point étant assimilée à un cercle.

Le principal inconvénient de cette méthode est le suivant : la courbe $F_{1}(x)$ ainsi que les courbes $F_{2}(x)$ et $F_{3}(x)$ passent par définition par les points parfaitement définis jusqu'au niveau $4-4,5 \mathrm{~m}$. Au-delà, on est obligé d'extrapoler jusqu'au niveau de $6 \mathrm{~m}$. On constate une déviation entre les courbes $F_{1}(x)$ d'un côté et $F_{2}(x), F_{3}(x)$ de l'autre, surtout pour les catégories de petits écarts à la verticale.

Les valeurs des coefficients des équations de chaque arbre ont été déterminés [9]. 


\section{Simulation du sciage}

3.1. Positionnement de la grume sur le chariot de scie de tête

Les grumes réelles sont placées telles que le plan xoy (qui contient les verticales de mesures) considéré précédemment soit parallèle au plan de coupe. Afin de déterminer la section des produits ayant la longueur maximale de chaque billon, le programme calcule avec précision la flèche présentée par la grume (fig. 3 : TAPOK), ce qui nous permet de trouver la section la plus haute de la grume sur le banc de sciage, ainsi que sa position par rapport au fin bout (AAFB).

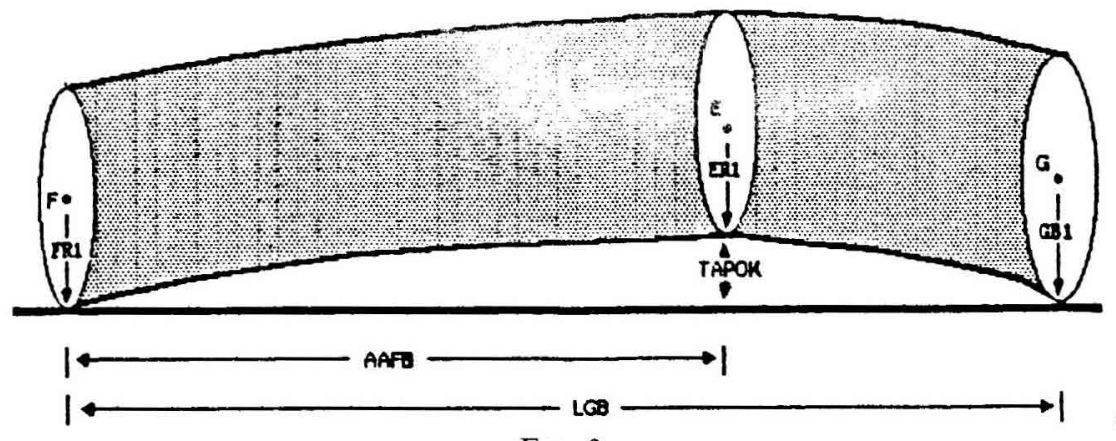

Fig. 3

Positionnement de la grume sur le chariot. Log position on the headsaw carriage.

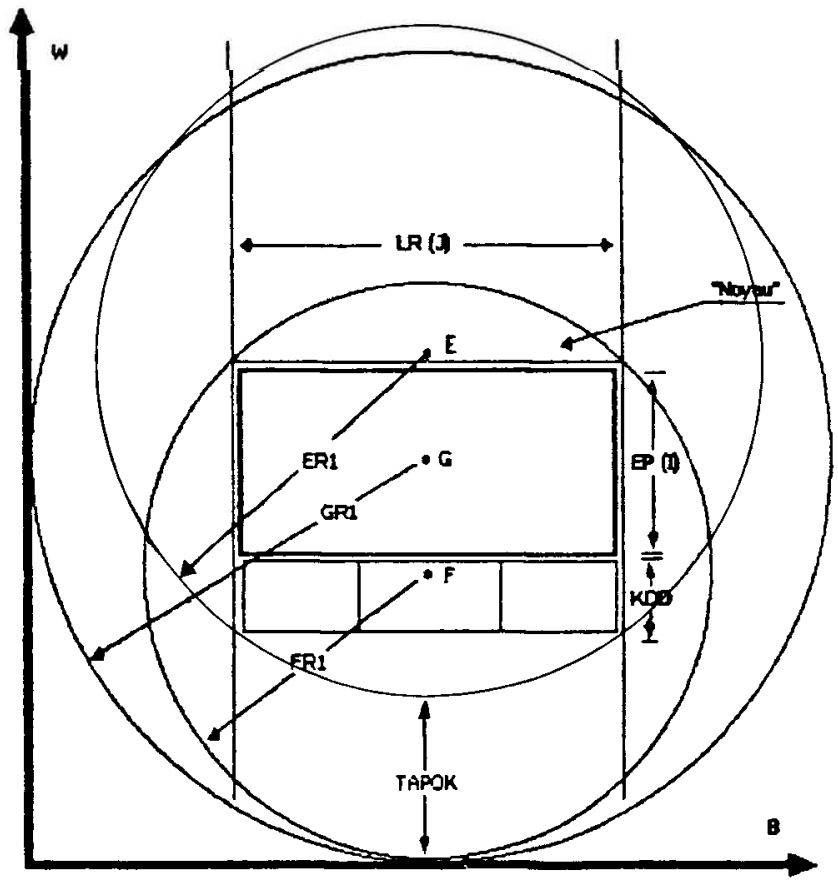

FIG. 4

Produits dans le «noyau». Products in the cant. 
La grume vue de bout (fig. 4) présente donc trois sections représentatives : fin bout (F, FR1), plus haute section (E, ER1) et gros bout (G, GR1). Les produits de plus grande longueur qu'on pourra tirer, doivent donc appartenir à l'intersection de ces trois cercles.

A la suite de différentes discussions avec les professionnels, quatre possibilités de tronçonnage à des hauteurs différentes, compte tenu des longueurs des produits à tirer, ont été retenues. Ces découpes sont :

- charpente (6 m environ);

$-3 \mathrm{~m}$ ( $1^{* \mathrm{r}}$ billon), plus le reste $\left(2^{*}\right.$ billon);

$-2,60 \mathrm{~m}\left(1^{\mathrm{er}}\right.$ billon), plus le reste ( $2^{\prime \prime}$ billon);

- $2.40 \mathrm{~m}$ ( $1^{\circ \mathrm{r}}$ billon), plus le reste $\left(2^{\prime \prime}\right.$ billon).

\subsection{Optimisation du sciage}

Le tableau 1 des produits à obtenir a été établi avec les scieurs landais et à partir du tableau des sections standards du pin maritime.

\section{TABLEaU 1}

Dimensions et prix des produits finis.

Dimensions and prices of final products.

\begin{tabular}{|c|c|c|c|}
\hline Usage & $\begin{array}{l}\text { Longueur à } \\
\text { tester (m) }\end{array}$ & $\begin{array}{c}\text { Sections à } \\
\text { tester }(\mathrm{mm})\end{array}$ & $\begin{array}{c}\text { Valeur } \\
\text { indicative }\end{array}$ \\
\hline $\begin{array}{l}\text { Maison à } \\
\text { ossaturo } \\
\text { en bois }\end{array}$ & $\begin{array}{l}2,40(1) \\
2,60 \\
3,00\end{array}$ & $40 * 100$ & $800 \mathrm{~F} / \mathrm{m}^{3}$ \\
\hline Charpente & $\begin{array}{c}\text { environ } \\
6,00\end{array}$ & $\begin{array}{l}220 * 80 \\
240 * 100 \\
260 * 100\end{array}$ & $900 \mathrm{~F} / \mathrm{m} 3$ \\
\hline Palette & 2,40 & $22 * 145$ & $700 \mathrm{~F} / \mathrm{m} 3$ \\
\hline $\begin{array}{l}\text { Produits } \\
\text { en toutes } \\
\text { longueurs } \\
\text { (II) }\end{array}$ & $>2,40$ & $27-40 * \mid \begin{array}{l}80 \\
100 \\
120 \\
145 \\
180\end{array}$ & $800 \mathrm{~F} / \mathrm{m}^{3}$ \\
\hline
\end{tabular}

(I) Ces dimensions s'étendent à la livraison en avivés séchés. Pour la découpe à l'état vert on ajoute les 5 p. 100 de perte occasionnés par le séchage sur toutes les dimensions.

(II) Le courson se fait sur 1,50 et est estimé à $550 \mathrm{~F} / \mathrm{m}^{3}$. 


\subsection{Produits charpente (environ $6 \mathrm{~m}$ )}

On essaie d'abord de tirer le nombre maximal de produits de $6 \mathrm{~m}$ dans le «noyau» (intersection de trois certes sur la figure 4). Dans ce cas, on constate que la seule possibilité de débit est de les placer horizontalement, contrairement aux autres cas. La partie du noyau qui reste (KDD en figure 4) est optimisée en utilisant deux fois des produits de $3 \mathrm{~m}$ de longueur.

La combinaison qui donne le plus de produits de longueur maximum constitue le point de départ pour l'optimisation des dosses.

Après le débitage du noyau, il reste 5 dosses ( 3 dosses longues et deux courtes, ces dernières provenant du côté concave). Les dosses de deux côtés et la dosse supérieure sont tronçonnées au niveau de la section la plus haute, ce qui donne en fait 8 dosses (fig. 5), assimilées à des parties de troncs de cône (fig. 6).

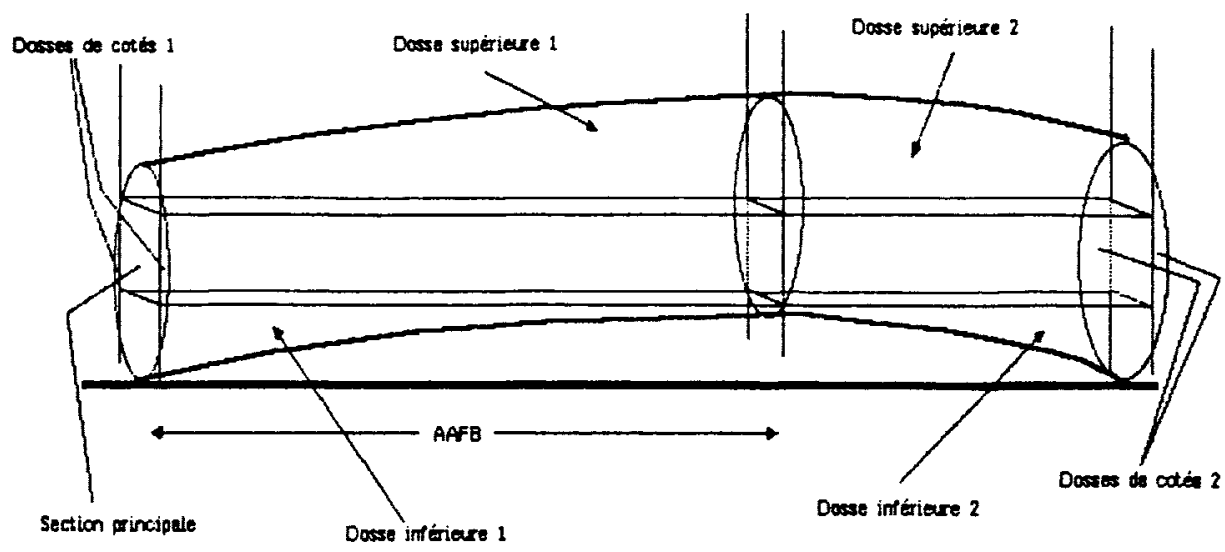

FIG. 5

Formation des dosses.

Slab formation.

A partir du calcul de l'intersection de plans parallèles avec la partie de tronc de cône, on définit successivement l'épaisseur, la largeur et la longueur du produit à tirer qui sont d'ailleurs placés parallèlement à la surface obtenue lors du débitage du noyau.

\subsection{Produits de plus petites longueurs}

On positionne les billons de plus petites longueurs sur le chariot, de la même façon que précédemment, et après la recherche des variables représentatives (TAPOK. $A A F B, E R 1)$ ils sont débités sur quartelot.

Le premier produit de côté est d'abord choisi. En effet, l'expérience des scieurs et les études sur l'optimisation $[3,4,5,10]$ montrent que le premier trait de scie réalisant le premier découvert a une importance primordiale et conditionne le rendement matière. 

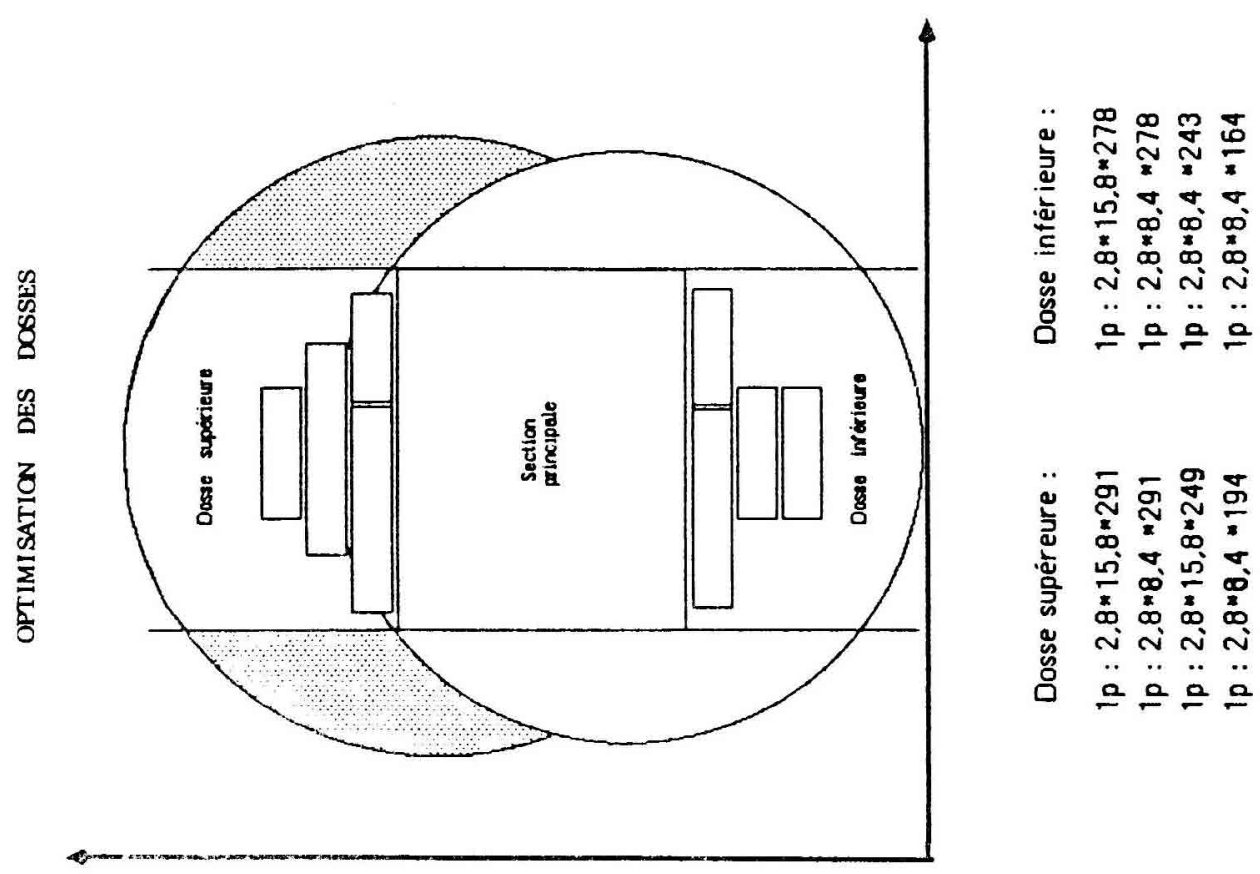

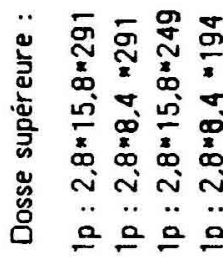

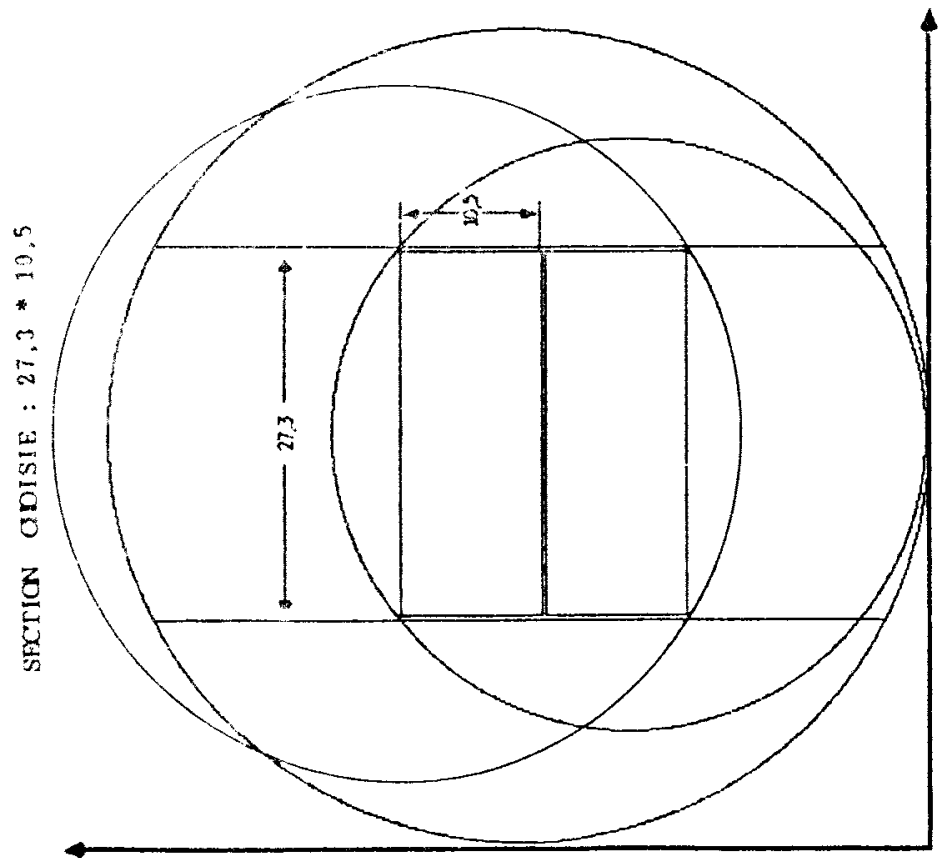

(2)

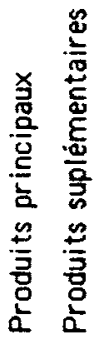



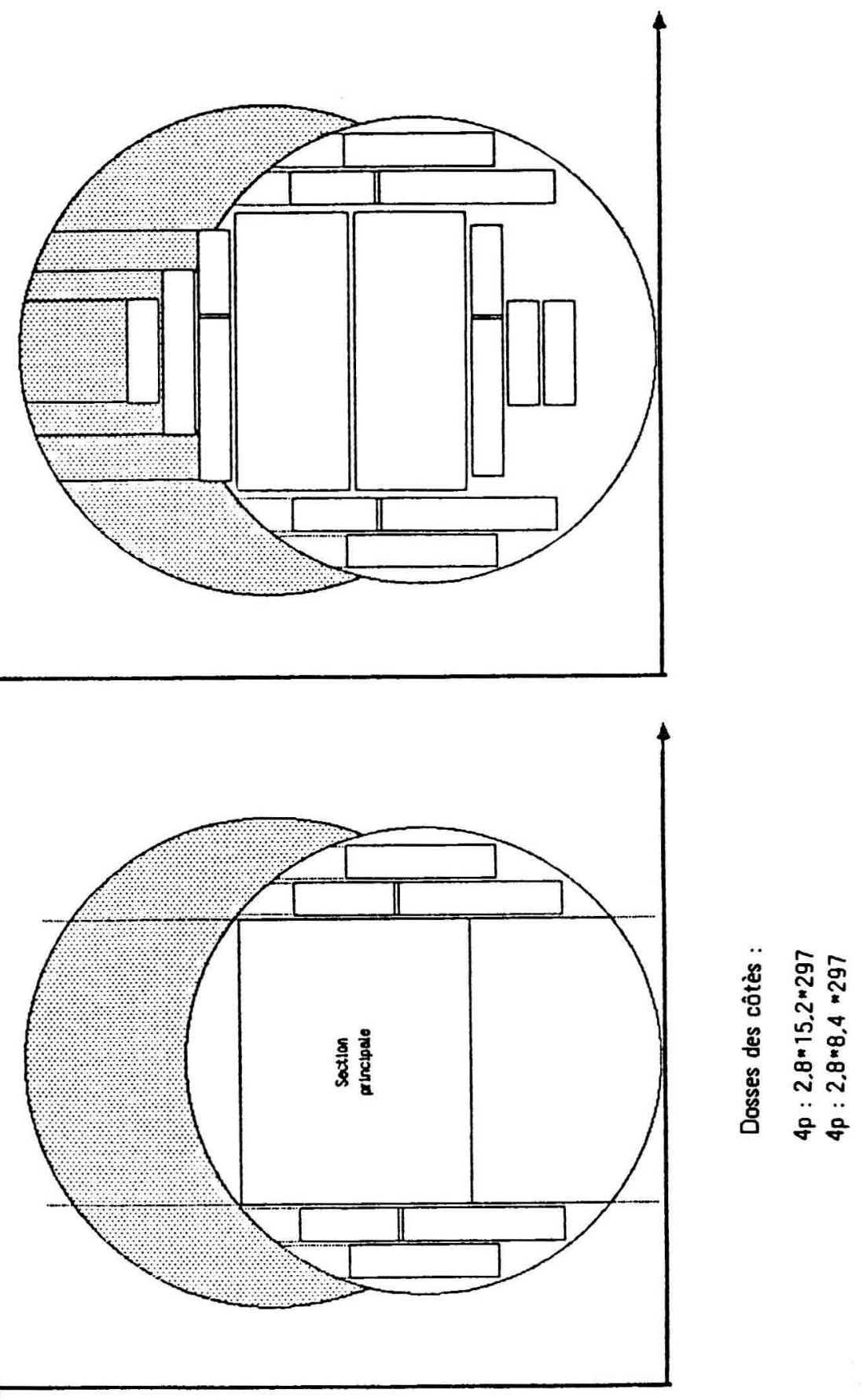

0
$\dot{0}$
$\dot{1}$ 
Le calcul du placement de ce premier trait étant réalisé, on simule ensuite le débit des plateaux que l'on peut placer dans le noyau en utilisant successivement les différentes largeurs et en tenant compte de ce que l'on peut tirer des dosses.

Ce calcul est réalisé pour chaque proposition de tronçonnage (tabl. 2 et fig. 7 ).

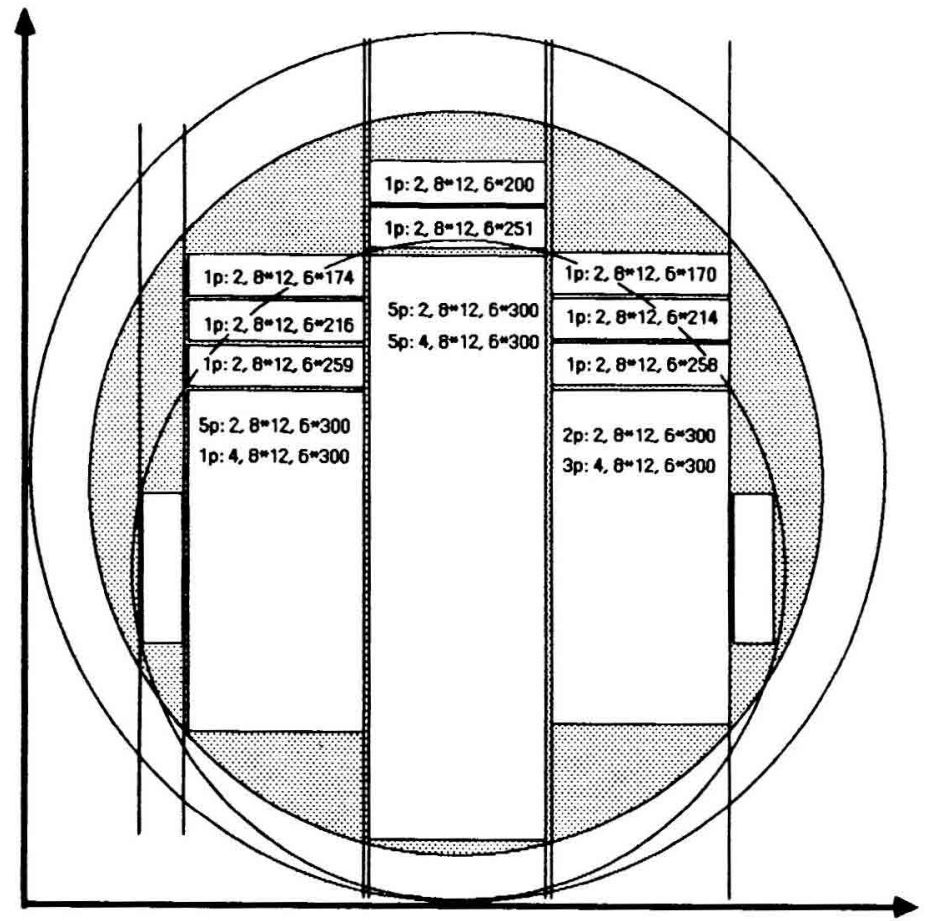

FIG. 7

Produits des plus petites longueurs (en $\mathrm{cm}$ ). Products of the smallest lengths.

Billon de 3,02 m (Proposition choisie).

Remarque : La section dite «la plus haute» de ce billon ne correspond pas strictement à celle définie aux figures 3 et 4 , à cause de la particularité de sa forme (ici convexe dans la partie inférieure, rectiligne dans la partie supérieure).

\section{Résultats}

\subsection{Influence de la longueur des produits}

Le rendement matière des produits des longueurs les plus petites est toujours plus grand que celui obtenu pour les longueurs les plus grandes. En ne prenant en compte que les produits principaux, le volume obtenu après tronçonnage est presque le triple de celui obtenu sur toute la longueur (rapport $1 / 2,8$ à $1 / 3$ ). Ce 


\section{TABleau 2}

Programme courbe.

Run-listing example.

Donnez les paramètres de votre arbre en $\mathrm{cm}$.

Ev. convexe $0=? 113$

Ev. convexe $150=$ ? 77

Ev. convexe $266=? 51$

Diamètre gros bout $1^{\text {er }}$ billon, $\mathrm{GD} 1=$ ? 61

Diamètre fin bout $1^{\text {er }}$ billon, $\mathrm{PD} 1=$ ? 50

Diamètre fin bout $2^{\mathrm{e}}$ billon, DD1 $=$ ? 47

Longueur $2^{e}$ billon, L2B $=$ ? 205

Les coefficients des courbes :

Côté convexe :
$\mathrm{A} 1=-7.77319 \mathrm{E}-05$
$\mathrm{B} 1=.253759$
$\mathrm{C} 1=0$

Milieu géométrique :

$\mathrm{A} 2=-1.20398 \mathrm{E}-04$

$\mathrm{B} 2=.292474 \quad \mathrm{C} 2=-31.7504$

Côté concave :
$\mathrm{A} 3=-1.57879 \mathrm{E}-04$
$\mathrm{B} 3=.33173$
$\mathrm{C} 3=-64.0677$

ESSAI POUR LES PRODUTS DE LONGUEUR MAX.

Les caractéristiques de votre billon sont :

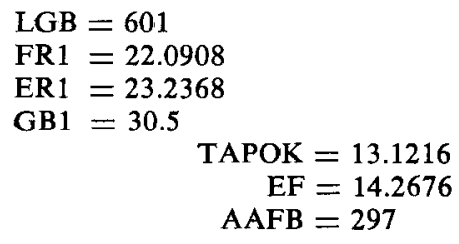

Volume max. de la première section : TV6 $=0.344553$

Nombre des produits : TAR $=2$

Largeur choisie $\quad: \operatorname{LR}(\mathbf{J})=27.3$

Optimisation des dosses

Nombre des produits pour chaque dosse $=$ TAR

Dosse supérieure 1: TAR $=6$

Dosse inférieure 1: TAR $=10$

Première dosse des côtés : TAR $=13$

Deuxième dosse des côtés : TAR $=16$

Dosses arrières:

Dosse supérieure 2: TAR $=25$

Dosse inférieure 2: TAR $=29$

Première dosse des côtés $2:$ TAR $=36$

Fin d'optimisation : TAR $=43$ 


\section{ESSAI POUR LES PRODUITS DE LONGUEUR $242 \mathrm{~cm}$}

$\mathrm{XF} 24(3)=247.403$

YF24 (3) $=8.33988$

Les caractéristiques de votre billon sont:

$\mathrm{LGB}=242$

$\mathrm{FR} 1=24.2799$

$\mathrm{ER} 1=26.9059$

$\mathrm{GB} 1=30.5$

$$
\begin{aligned}
\text { TAPOK } & =2.03562 \\
\text { EF } & =4.66163 \\
\text { AAFB } & =120
\end{aligned}
$$

Vol. max. obtenu, VOL $=.374267$

Largeur choisie, LR $(\mathrm{TJ})=12.6$

\section{RESTE DU BILLON $242 \mathrm{~cm}$}

Les caractéristiques de votre billon sont :

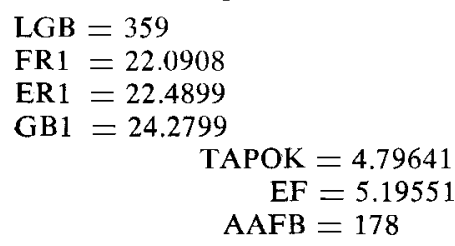

Vol. max. obtenu, VOL $=.378976$

Largeur choisie, LR (TJ) $=8.4$

Volume de cette proposition $=.753242$

\section{ESSAI POUR LES PRODUITS DE LONGUEUR $262 \mathrm{~cm}$}

Les caractéristiques de votre billon sont:

$$
\mathrm{O} \quad \mathrm{O} \quad \mathrm{O}
$$

Le volume de votre billon est 1.31869

Les volumes et les rendements obtenus pour chaque proposition de tronçonnage sont :

- Produits principaux charpente

$$
\begin{array}{lr}
\text { Volume }\left(\mathrm{m}^{*}\right) & \text { Rendement } \\
=.699848 & 53.07 \\
=.753242 & 57.12 \\
=.745572 & 56.53 \\
=.795435 & 60.32
\end{array}
$$

Rendement $(\%)$

- Billon de 2.42 plus le reste

- Billon de 3.02 plus le reste 
rapport diminue lorsque l'on considère en plus les dosses et il est alors compris entre $1 / 1,2$ et $1 / 1,5$. Cette diminution est due essentiellement au fait que la préférence du mode du sciage est la longueur des produits et non pas le rendement volumique.

Généralement de grands diamètres sont obtenus dans les classes de grands écarts à la verticale, mais le volume utilisable pour les produits de $6 \mathrm{~m}$ de longueur reste inférieur à ceux des classes des plus petits écarts : on ne peut même pas tirer de produits de charpente dans la classe d'écart supérieur à $60 \mathrm{~cm}$.

$\mathrm{Si}$ on prend en compte le rendement financier, le rapport est encore plus important à cause de la faible différence des prix fournis $\left(800 \mathrm{~F} / \mathrm{m}^{3}-900 \mathrm{~F} / \mathrm{m}^{3}\right.$, tabl. 1).

\subsection{Comparaison entre classes (tableau 3)}

Bien que le volume brut augmente généralement avec les classes d'écart à la verticale, on obtient le rendement maximum pour la classe de $40-49 \mathrm{~cm}$, résultat qui est en accord avec l'ordre des valeurs obtenues après le sciage du sous-échantillon. Par exemple, on constate que le volume moyen de la classe $50-59 \mathrm{~cm}$ est nettement supérieur à celui de la classe $30-39 \mathrm{~cm}$ mais que son rendement maximal après tronçonnage est inférieur.

TABLEAU 3

Comparaison entre classes.

Comparison between classes.

\begin{tabular}{|c|c|c|c|c|c|}
\hline \multirow{2}{*}{$\begin{array}{l}\text { Catégorie } \\
(\mathrm{cm})\end{array}$} & \multirow{2}{*}{$\begin{array}{c}\text { Volume } \\
\text { moyen }\left(\mathrm{m}^{3}\right)\end{array}$} & \multicolumn{4}{|c|}{ Rendement (\%) } \\
\hline & & $\begin{array}{l}\text { Prod. } \\
\text { charpente }\end{array}$ & $\begin{array}{l}\text { Prod. de } 2,4 \mathrm{~m} \\
\quad+\text { le reste }\end{array}$ & $\begin{array}{l}\text { Prod. de } 2,6 \mathrm{~m} \\
\quad+\text { le reste }\end{array}$ & $\begin{array}{l}\text { Prod. de } 3,0 \mathrm{~m} \\
\quad+\text { le reste }\end{array}$ \\
\hline $10-19$ & 0,5952 & - & 56,15 & 54,07 & 55,67 \\
\hline $20-29$ & 0,7630 & 43,25 & 54,89 & 54,63 & 56,44 \\
\hline $30-39$ & 0,8748 & 44,66 & 56,01 & 55,94 & 57,05 \\
\hline $40-49$ & 0,9056 & 50,62 & 56,89 & 57,89 & 57,83 \\
\hline $50-59$ & 0,9670 & 47,60 & 51,95 & 53,31 & 56,38 \\
\hline 60 et + & 0,8190 & - & 48,53 & 50,78 & 52,28 \\
\hline
\end{tabular}




\subsection{Analyse des résultats}

Dans une même classe, la solution optimale dans la majorité des cas est donnée par la solution du tronçonnage à $3 \mathrm{~m}$, due à la configuration de la partie concave.

Le rendement est en rapport avec le volume (dimensions) et la forme de chaque grume. Une grume avec un volume supérieur tend à avoir un rendement supérieur mais l'influence de la forme reste toujours prépondérante et susceptible d'inverser entièrement les résultats.

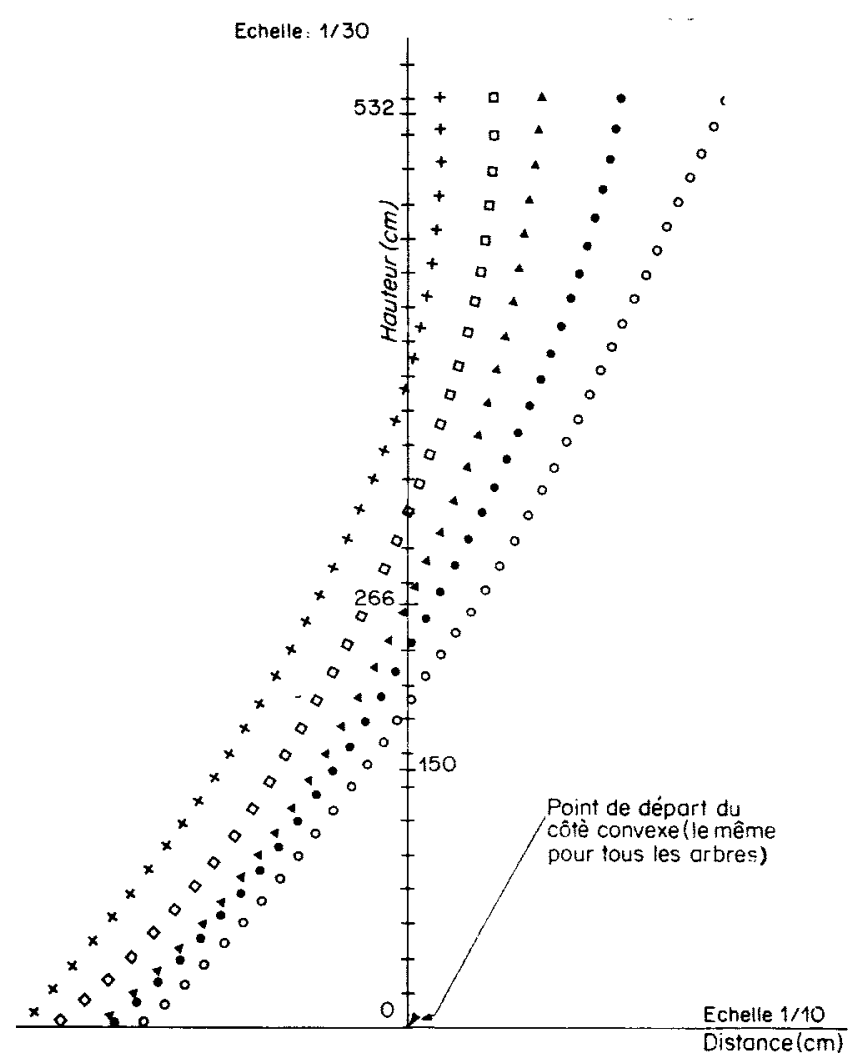

Fig. 8

Représentation des côtés concaves.

Catégorie : $50-59 \mathrm{~cm}$.

Concave sides representation.

$++t$ Arbre $n^{0} 131$.

000 Arbre $\mathrm{n}^{\circ} 133$.

- Arbre $\mathrm{n}^{\circ} 138$.

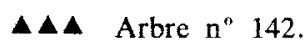

$\square \square \square$ Arbre $n^{\circ} 143$. 
De plus, aucune corrélation n'a pu être obtenue entre les rendements matière et l'écart à la verticalité. Aussi, on obtient le rendement maximum pour la classe $40-49 \mathrm{~cm}$, puis en second pour la classe $30-39 \mathrm{~cm}$ et en troisième pour la classe 50-59 cm. Cela ne signifie pas que les arbres penchés donnent un rendement meilleur que les droits mais l'influence simultanée du volume et de la forme produisent ce phénomène.

Les rendements calculés sont inférieurs aux rendements matière estimés sur le sous-échantillon scié. Mais cette comparaison n'est pas significative car les essais en scierie ont été réalisés sur des billons de $2 \mathrm{~m}$, et seulement pour les premiers $4 \mathrm{~m}$ de chaque grume. Par contre, nos calculs correspondent à des grumes de $6 \mathrm{~m}$ et les produits sont de plus grande longueur.

En ce qui concerne le rendement financier, on peut constater que la faible différence de prix entre charpente et produits de plus petites longueurs ne permet pas de privilégier les grandes longueurs.

A l'intérieur de chaque classe on constate une grande variabilité de la forme des grumes. Les arbres sont classés par rapport à l'écart à la verticale à $1,50 \mathrm{~m}$ mais ce classement n'a pas un intérêt mathématique comparable à son intérêt biologique (par exemple : l'amélioration génétique) pour plusieurs raisons :

a) Dans la plupart des cas, l'écart à la verticale à $1,5 \mathrm{~m}$ est beaucoup moins variable qu'à d'autres hauteurs, supérieures mais inférieures aussi (fig. 8). Cet accident de la forme qui affecte la grume sur toute sa longueur présente une divergence assez sensible surtout au-dessus de $1,50 \mathrm{~m}$.

b) Les mesures effectuées montrent une variabilité considérable des écarts à la verticale de la partie convexe des arbres de la même classe. Aussi la diminution des diamètres entre le niveau 0 et les niveaux supérieurs dans le même arbre, suit un rythme très varié pour les différents arbres de chaque classe. Ces deux points d'ailleurs (écarts du côté convexe et diamètres perpendiculaires) constituent des éléments prédominants pour la configuration de la forme des billons.

c) L'influence simultanée des points a) et b), précédemment décrits, a comme conséquence une très grande variation sur la flèche de la courbure qu'on estime avant d'optimiser chaque billon et qui définit le rendement matière de ceux-ci.

\section{Conclusion}

Du point de vue géométrique, une corrélation n'a pu être obtenue entre le rendement matière et l'écart à la verticalité. La faible différence de valeur entre les produits de grande longueur et les plus courts ne permet pas de justifier la recherche de produits longs. Cependant le rendement matière géométrique nous apparâit toujours comme insuffisant pour établir le classement de grumes courbes : la présence de bois de compression est un facteur dont il faudrait tenir compte, à cause de ses répercussions sur le comportement du bois débité, au séchage par exemple.

D'autre part, on a pu constater que l'écart à la verticale à $1,5 \mathrm{~m}$, malgré son intérêt scientifique, ne constitue pas un critère représentatif de la forme générale des grumes en vue de leur débit.

Rappelons que cette recherche n'est qu'une partie d'une étude plus générale sur le défaut de rectitude basale du pin maritime développée par le C.E.M.A.G.R.E.F. 


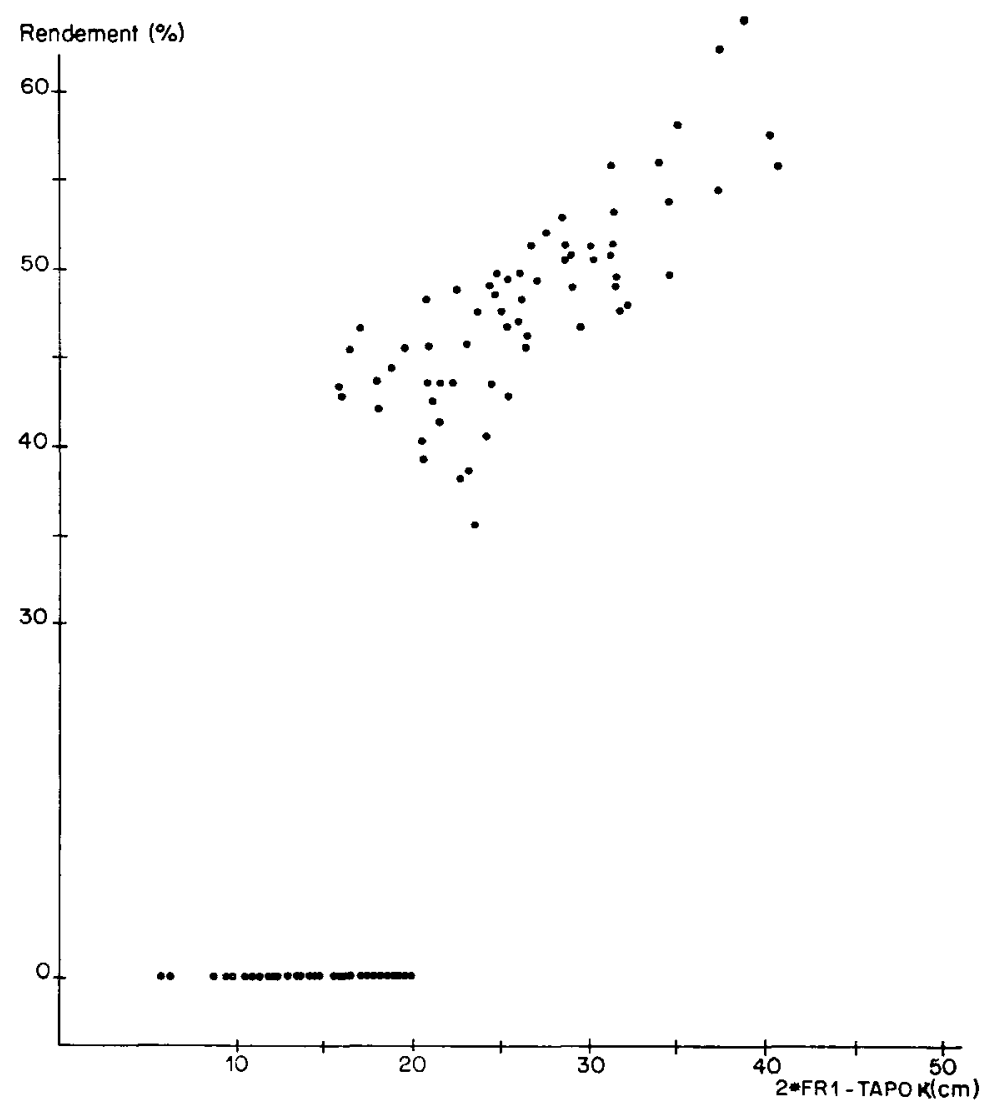

FIG. 9

Corrélation entre le rendement et le paramètre $H=2^{*} F R$ 1-TAPOK

Correlation between recovery and $H=2 * F R 1-T A P O K$.

Des simulations ont été également entreprises sur l'ensemble des 150 arbres sélectionnés, les diamètres sous écorce étant déterminés à partir de la mesure du périmètre et l'estimation de l'épaisseur de l'écorce obtenue par extrapolation des mesures effectuées sur le sous-échantillon.

Des résultats comparables, compte tenu des approximations, sont obtenus [9]. De nouveaux critères de classement ont été recherchés pour corréler le rendement matière d'une manière plus significative et la grandeur $\mathrm{H}=2^{*} \mathrm{FR} 1$ - TAPOK (diamètre fin bout - flèche) est alors apparue comme la plus satisfaisante (fig. 9). La figure 9 montre qu'au-dessus d'une valeur limite du paramètre $\mathrm{H}$ de l'ordre de $22 \mathrm{~cm}$ il est toujours possible de scier des produits de $6 \mathrm{~m}$. Entre cette valeur et $16 \mathrm{~cm}$, on obtiendra ou non de tels produits suivant la forme de la grume.

Reçu le 2 juillet 1985.

Accepté le 10 février 1986. 


\section{Summary \\ Sawing optimization of maritime pine}

This study is a part of a larger one, C.E.M.A.G.R.E.F. co-ordinated programm, concerning the exploitation of maritime pine (Pinus pinaster) of Landes, France. The major particulary of this species is its curved trunk. Thanks to collected data on a sample of 150 trees, a model of the form of these trees, based on two parabolas was taken out. These trees were distributed in 6 categories according to their deviation from the vertical axis. This deviation was measured at $1.50 \mathrm{~m}$ high on the concave side.

Until now, these trees have been converted into 2 and $2.6 \mathrm{~m}$ length logs. Looking for better utilisation as construction lumber, the sawing simulation was made on $6,3,2.6$ and $2.4 \mathrm{~m}$ length logs.

From the geometrical point of view, we did not find any correlation between lumber recovery and vertical deviation. Some new classification criteria have been tested, to correlate the volume recovery more significantly. The value $\mathrm{H}=$ (Small diameter-curve height) seems to be the most satisfactory. Morever, the compression wood would be an additional parameter to be taken into consideration to the final $\log$ classification, because of its influence on the lumber quality. wood.

Key words : Sawmilling, sawing optimization. Pinus pinaster, curved logs, compression

\section{Références bibliographiques}

1. Bouzinekis A., 1984. Optimisation du sciage du pin maritime, D.E.A. Sciences du Bois, Nancy, octobre 1984.

2. Centre technique du bois, 1983. Optimisation du débit d'avivés. C.T.B., Courrier de l'industrie du bois et de l'ameublement, juillet 1983.

3. Centre technique du bois, 1983. Optigrum. Fonctionnement du système. C.T.B., juin 1982.

4. Goulet M., Gautier R., Lazole J.C., 1975. Essai de simulation du débitage à arêtes vives dans les conifères de faible diamètre. Univ. Laval, Québec, 12/1975.

5. Hallock H., Lewis D., 1978. Increasing softwood dimension yield from small logs. For. Pr. Lab., Madison, Wis., 1978.

6. Hallock H., Lewis D., 1974. Best opening face program (B.O.F.). Austr. For. Ind. Journal, November 1974.

7. Keller R., Mosnier J.C., 1984. Verticalité et vigueur du pin maritime. Rapport de fin de contrat D.G.R.S.T.-C.E.M.A.G.R.E.F. Laboratoire de Recherches sur les Produits Forestiers. C.N.R.F.-E.N.G.R.E.F., Nancy, 1985 (14 pages).

8. Lepagf F., Martin P., Ris G., 1981. Optimisation en temps réel du tronçonnage de planches. Le Nouvel Automatisme, octobre 1981.

9. Martin P., BouZinekis A., 1984-1985. Rapports d'avancement des travaux. Rapport de fin de contrat D.G.R.S.T.C.E.M.A.G.R.E.F., 1984-1985.

10. Pariat Y. Optimisation de découpe de grumes coniques. D.E.A. Sciences du Bois, Nancy, octobre 1983.

11. Richardos D., Adkins W., Hallock H., Bulgrin E., 1979. Simulation of hardwood log sawing. For. Pr. Lab., Madison, Wis., 1979.

12. Savsar M., Kersavage P., 1982. Mathematical model for determining the quantity of materiales produced in sawmilling. For. Pr. Journal, December 1982. 